\title{
Leveraging medical context to recommend semantically similar terms for chart reviews
}

\author{
Cheng Ye ${ }^{1 *}$, Bradley A. Malin ${ }^{1,2,3}$ and Daniel Fabbri, ${ }^{1,2}$
}

\begin{abstract}
Background: Information retrieval (IR) help clinicians answer questions posed to large collections of electronic medical records (EMRs), such as how best to identify a patient's cancer stage. One of the more promising approaches to IR for EMRs is to expand a keyword query with similar terms (e.g., augmenting cancer with mets). However, there is a large range of clinical chart review tasks, such that fixed sets of similar terms is insufficient. Current language models, such as Bidirectional Encoder Representations from Transformers (BERT) embeddings, do not capture the full nontextual context of a task. In this study, we present new methods that provide similar terms dynamically by adjusting with the context of the chart review task.
\end{abstract}

Methods: We introduce a vector space for medical-context in which each word is represented by a vector that captures the word's usage in different medical contexts (e.g., how frequently cancer is used when ordering a prescription versus describing family history) beyond the context learned from the surrounding text. These vectors are transformed into a vector space for customizing the set of similar terms selected for different chart review tasks. We evaluate the vector space model with multiple chart review tasks, in which supervised machine learning models learn to predict the preferred terms of clinically knowledgeable reviewers. To quantify the usefulness of the predicted similar terms to a baseline of standard word2vec embeddings, we measure (1) the prediction performance of the medical-context vector space model using the area under the receiver operating characteristic curve (AUROC) and (2) the labeling effort required to train the models.

Results: The vector space outperformed the baseline word2vec embeddings in all three chart review tasks with an average $A U R O C$ of 0.80 versus 0.66 , respectively. Additionally, the medical-context vector space significantly reduced the number of labels required to learn and predict the preferred similar terms of reviewers. Specifically, the labeling effort was reduced to $10 \%$ of the entire dataset in all three tasks.

Conclusions: The set of preferred similar terms that are relevant to a chart review task can be learned by leveraging the medical context of the task.

Keywords: Electronic medical records, Data science, Chart reviews, Clinically similar terms, Vector space model

\section{Background}

In a clinical chart review task [1], a clinically knowledgeable person (e.g., physician, medical student, or nurse) combs through electronic medical records (EMRs) [2-4]

\footnotetext{
*Correspondence: cheng.ye.1@vumc.org

1 Department of Computer Science, Vanderbilt University, 2301 Vanderbilt

Place, PMB 351679, Nashville, TN 37235-1679, USA

Full list of author information is available at the end of the article
}

for specific data of interest. Chart reviews are time-consuming and costly because a patient's chart may be composed of hundreds of clinical notes. Various automated approaches have been developed to improve the efficiency of chart reviews. A particularly promising information retrieval (IR) method to assist with chart reviews is query expansion [5-8]. This method expands the original search terms into a set of similar terms and, subsequently, returns medical notes that contain at least one 
of the expanded terms. In addition, these similar terms can be applied to highlight text within a note and assist the reviewer to identify the important snippets of text quickly [9-14].

Chart reviews are relied upon to answer a wide range of questions-from determining the current stage of cancer for a particular patient to identifying which drugs appear to be most ordered for the treatment of seizures. These different chart review tasks can be assisted by query expansion methods; however, given the range of chart review tasks that derive from a single search term, a static set of similar terms is not appropriate for all tasks. Rather, the set of similar terms should adjust based on the context of the chart review task. For example, a reviewer looking for an epilepsy diagnosis likely cares more about EEG results, while a reviewer looking at medications for treating epilepsy likely cares more about indications of the drug Keppra. Therefore, the set of similar terms should dynamically adjust based on the task and context of the review.

To date, natural language processing methods for term similarity, such as word2vec $[15,16]$ and more recently Bidirectional Encoder Representations from Transformers (BERT) [17], provide embeddings to capture term similarity that can be used to recommend terms for expansion. For example, these methods now support dynamic query refinement in the Google search engine $[17,18]$. Importantly, the similarity between two words within an embedding depends on the training data set used to build the embedding[19], as well as the data set used to fine-tune the model (e.g., refining BERT into BioBERT [20]). Thus, as the training or finetuning data set is changed, the set of expansion terms similarly will change.

While word2vec model training and finetuning activities modify word similarities according to textual relationships, there are a number of ways that clinical documentation can be influenced by factors not explicitly documented in the text. For example, word choice can be modified by a number of factors, including, but not limited to, who authored the note, the section in which the word is documented, or the age of the patient. Similarly, when reviewing charts, these different usages impact the information needed for a chart review. In this research, we investigate how such contextual information can be leveraged to modify term similarity for chart review tasks.

In this paper, we introduce a medical-context vector space, which corresponds to a collection of the usage frequencies of clinical terms in various real-world medical situations, to identify task-appropriate similar terms. We evaluate the medical-context vector space for prediction of preferred similar terms in chart review tasks for acute myocardial infarction (AMI), Crohn's disease, and diabetes. Each of these tasks is notable in that they consist of complex requirements for identifying similar terms for chart reviews, including terms for relevant diagnoses, medications, findings, and history. Additional file 1: Table A, Table B, and Table C demonstrate the 10 most similar terms for "Crohn," "Acute Myocardial Infarction (AMI)," and "Diabetes." It can be seen that there are similar terms in common across the various medical contexts, as well as specific similar terms for certain medical contexts. For example, as shown in Additional file 1: Table A, "ileitis" and "ileum" are commonly used similar terms for "Crohn", but "pancolitis" is only used in the outpatientvisit note types from the gastroenterology department, which implies that the system will recommend "pancolitis" to users only when they focus on reviewing a specific note type from a certain department.

\section{Methods}

\section{Medical-context vector space}

To orient the reader, we provide a running example in Fig. 1, which depicts the medical context associated with a fictitious medical note. The note was created for a 26-year-old male patient by a physician in the NeuroEpilepsy Department. The Medical Context Type refers to the general context of a term's usage, the Medical Context refers to a specific type of data in the context, and the Attribute refers to the specific value. Our objective is to capture information regarding how terms are used in different medical contexts.

We identified four types of usage context types resulting in ten specific contexts from the EMR system [21] of Vanderbilt University Medical Center (VUMC):

(1) Hospital Organizational Structure. The role and speciality of the note's author (i.e., job titles and departments) based on the hospital's organizational structure.

(2) Medical Events. The documented diagnoses and procedures of a patient, including ICD-9/10 codes, CPT codes, and Emergency Department chief complaints that are documented around the time the note is written.

(3) Demographics. Patient gender (male, female, and unknown) and age (quantized into ten-year bins).

(4) Medical Note Structure. Clinical note types and sections.

These contexts represent commonly used descriptors of patient care and can be used to infer non-textual information regarding how terms are used in different situations. Leveraging this structure, we build the medical-context vector space through the following steps: 


\begin{tabular}{|l|l|l|}
\hline Medical Context Type & Medical Context & Example Attribute \\
\hline \multirow{2}{*}{$\begin{array}{l}\text { Hospital Organizational } \\
\text { Structure }\end{array}$} & Author Department & Neuro-Epilepsy \\
\cline { 2 - 3 } Demographics & Author Staff Title & Assistant Professor \\
\hline \multirow{4}{*}{ Medical Events } & Gender & Male \\
\cline { 2 - 3 } & Age & 26 \\
\hline \multirow{4}{*}{ Medical Note Structure } & ICD Events within 48 hours & $789.39,345.41, \ldots$ \\
\cline { 2 - 3 } & CPT Events within 48 hours & G0463,99214,... \\
\cline { 2 - 3 } & Chief Complaints within 48 hours & Seizure \\
\cline { 2 - 3 } & Note Type & Progress Note \\
\cline { 2 - 3 } & Tote Sections & History, Conclusion, ... \\
\hline
\end{tabular}

Fig. 1 The medical context for an example clinical note

(1) Preprocessing: First, we extract a subset of notes from the EMR system (e.g., all medical notes created in the year 2016). For each medical note and context, we extract the associated attribute values (as shown in Fig. 2) and filter out stop words (e.g., "a" and "of") and single-character words.

(2) Initialization: We define ten medical contexts $C=\left\{C_{1}, C, . ., C_{10}\right\}$ as shown in Table 1 . For each clinical term $\boldsymbol{w}$, we initialize its medical-context vector to all zeros:

$$
u_{w}=\left\{u_{c 1}(w), u_{c 2}(w), \ldots, u_{c 10}(w)\right\}=\left\{\overrightarrow{0}_{c 1}, \overrightarrow{0}_{c 2}, \ldots, \overrightarrow{0}_{c 10}\right\}
$$

(3) Accumulation: For each word, we increment its medical-context vector based on the occurrence of the word in each context. For example, in Fig. 2, we add four to the Neuro-Epilepsy dimension of the author's department medical context in the medical-context vector of $E E G$ if the author uses EEG four times. This step is repeated for each note.

(4) Normalization: Next, as shown in Fig. 3, we normalize the counts of clinical terms in each context into medical-context proportions $[0.0,1.0]$ (i.e., the medical context vectors). At the end of this process, each clinical term is represented as a medical-context vector that consists of its normalized frequencies in each medical context.

The medical-context vector represents how a term is used within a medical situation. We define the medical-context similarity of two clinical terms $w_{i}$ and $w_{j}$ in the medical context $C_{k}$ as the cosine similarity of their medical-context vectors:

$$
S_{c_{k}}\left(w_{i}, w_{j}\right)=\frac{u_{c_{k}}\left(w_{i}\right) \cdot u_{c_{k}}\left(w_{j}\right)}{\left\|u_{c_{k}}\left(w_{i}\right)\right\| \times\left\|u_{c_{k}}\left(w_{j}\right)\right\|}
$$

The similarity of two clinically similar terms in a medical context provides intuition into their semantic relationships. For example, as shown in Fig. 4, in the department medical context, the cosine similarity of diabetes and hypertriglyceridemia is 0.56 , which suggests that they are moderately similar in the department medical context, while other terms have a better similarity (e.g., hypothyroidism) in that context. For further illustration, Additional file 1: Table A, Table B and Table $\mathrm{C}$ report on the top similar terms in different medical contexts.

We define the medical-context similarity vector of two clinical terms $w_{i}$ and $w_{j}$ as a vector containing the medical-context similarities across all medical contexts. Each index of the vector is equal to the cosine similarity of each term's medical-context vector for one specific context:

$$
S\left(w_{i}, w_{j}\right)=\left\{S_{c 1}\left(w_{i}, w_{j}\right), S_{c 2}\left(w_{i}, w_{j}\right), \ldots, S_{c 10}\left(w_{i}, w_{j}\right)\right\}
$$

The medical-context similarity vector of two clinical terms represents their relationships across all medical contexts. For example, Fig. 5 shows the medical-context similarity vector of diabetes and hypertriglyceridemia. It can be seen that their similarity in the Note Type, Author Department, and Chief Complaints contexts are much lower than in other contexts. Therefore, if a reviewer prefers terms that have a similar distribution of medicalcontext frequencies as diabetes in the Note Type, Author Department, and Chief Complaints contexts, then the reviewer may not prefer hypertriglyceridemia.

Medical-context similarity vectors provide a weighted vector space, which can be used to identify which similar 


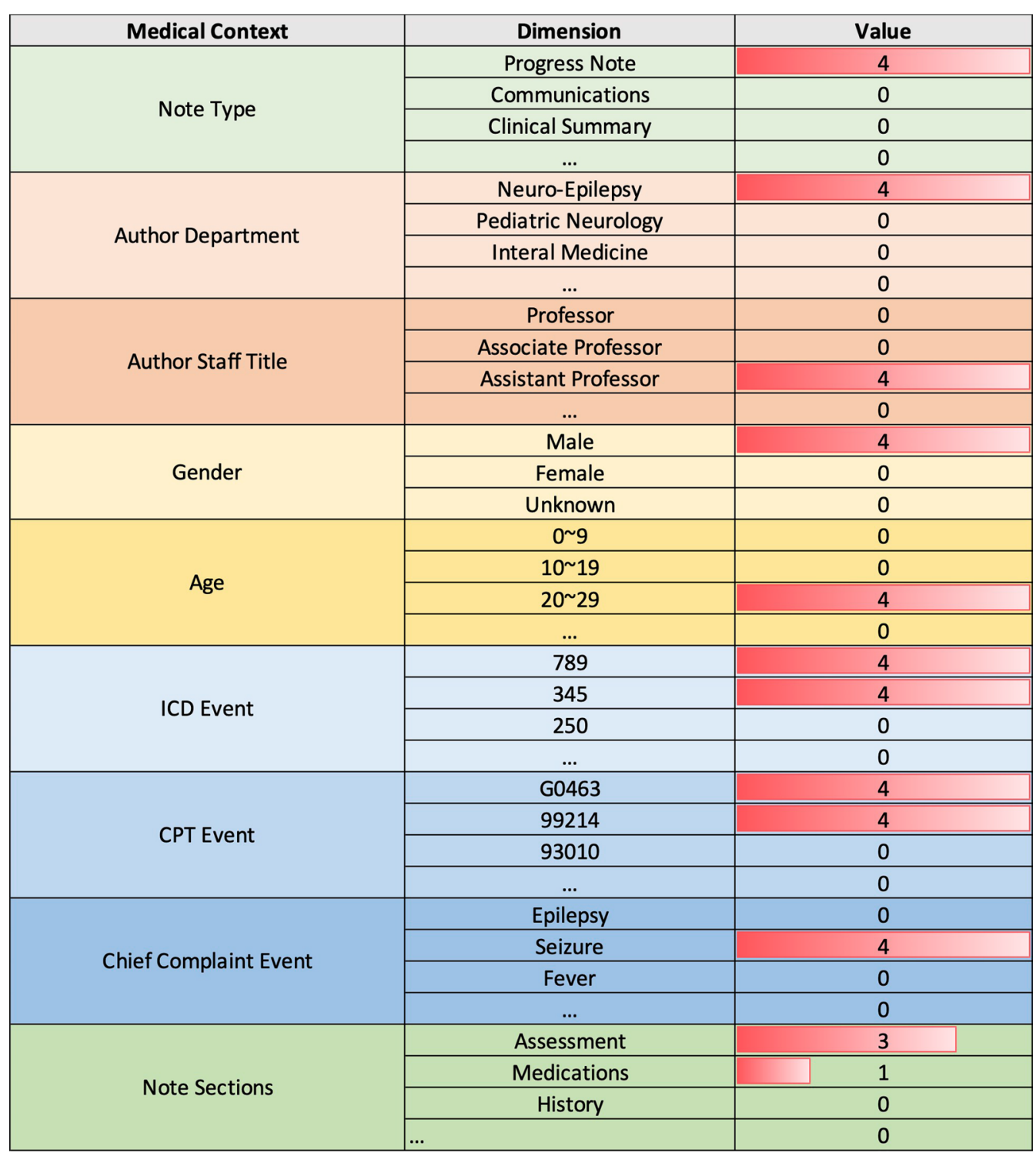

Fig. 2 The medical-context counts of EEG according to their context in the example note in Fig. 1

Table 1 The dimensions for clinical terms in each medical context

\begin{tabular}{llc}
\hline Context type & Medical context & Dimensions \\
\hline Hospital organizational structure & Departments & 258 \\
& Staff & 158 \\
Medical events & CPT events & 6537 \\
& ICD events & 957 \\
& Chief complaint events & 11,595 \\
Demographics & Age & 10 \\
Medical note structure & Gender & 3 \\
& Note type & 1514 \\
& Note section & 61 \\
& Top note section & 5 \\
\hline
\end{tabular}

terms are preferred for a specific chart review task. Thus, instead of providing chart reviewers with a static set of similar terms, the set can be adjusted as information is collected about the context of a task. This adjustment can be done in an online learning style where (i) a chart reviewer first inputs a keyword, (ii) is presented with a list of ranked similar terms, and (iii) the reviewer then starts the chart review task, in which the goal is to highlight text in notes that are evidence for answering clinical questions. Given the iteratively-gathered highlighted text as input, a supervised machine learning model for term prediction is iteratively trained after each input to capture the contexts that the reviewer deems to be most 


\begin{tabular}{|c|c|c|c|c|c|}
\hline Medical Context & Top 3 dimensions & Usage & ts by usage context & Usage & cies by usage context \\
\hline \multirow{3}{*}{ Note Type } & history \& physical - anesthesiology (pediatric anes) & & 4661 & & 0.19 \\
\hline & progress note - neurology & 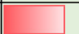 & 3398 & & 0.14 \\
\hline & administrative - prior authorization & 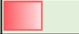 & 2170 & $\square$ & 0.09 \\
\hline \multirow{3}{*}{ Author Department } & Neuro-Epilepsy Division & & 18663 & & 0.21 \\
\hline & In-Patient Nurse Practitioners & & 10812 & 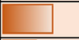 & 0.12 \\
\hline & VUH Nurse Practitioners & & 6901 & 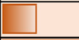 & 0.08 \\
\hline \multirow{3}{*}{ Author Staff Title } & Clinical Fellow & & 20163 & & 0.22 \\
\hline & Assistant Professor & & 14512 & 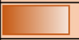 & 0.16 \\
\hline & Assistant In, NP Acute & & 11885 & 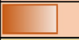 & 0.13 \\
\hline \multirow{3}{*}{ Gender } & $\mathrm{F}$ & & 12645 & & 0.5 \\
\hline & $M$ & & 12430 & & 0.5 \\
\hline & $U$ & & 3 & & 0 \\
\hline \multirow{3}{*}{ Age } & $0 \sim 9$ & & 6660 & & 0.27 \\
\hline & $10 \sim 19$ & $=$ & 3636 & 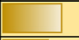 & 0.14 \\
\hline & $60 \sim 69$ & 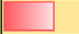 & 2777 & 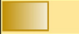 & 0.11 \\
\hline \multirow{3}{*}{ ICD Event } & 780 & 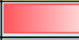 & 4735 & 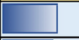 & 0.13 \\
\hline & 345 & 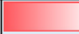 & 4265 & \begin{tabular}{|l} 
\\
\end{tabular} & 0.12 \\
\hline & V72 & $\square$ & 1792 & $\square$ & 0.05 \\
\hline \multirow{3}{*}{ CPT Event } & 80048 & & 6282 & 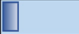 & 0.04 \\
\hline & G0463 & $=$ & 4678 & $\pi$ & 0.03 \\
\hline & 85027 & 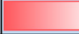 & 4543 & ] & 0.03 \\
\hline \multirow{3}{*}{ Chief Complaint Event } & OBSTRUCTIVE SLEEP APNEA (ADULT) (PEDIATRIC) & & 248 & $\square$ & 0.07 \\
\hline & EPILEPSY & & 224 & $\square$ & 0.07 \\
\hline & DENTAL CARIES & & 192 & $\square$ & 0.06 \\
\hline \multirow{3}{*}{ Note Section } & history_present_illness & 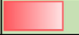 & 3315 & $\mathrm{E}$ & 0.16 \\
\hline & assessment & 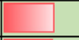 & 2814 & 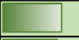 & 0.14 \\
\hline & findings & $\square$ & 2726 & $\square$ & 0.13 \\
\hline \multirow{3}{*}{ Top Note Section } & assessment & 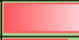 & 4838 & & 0.33 \\
\hline & medications & 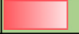 & 3573 & & 0.24 \\
\hline & findings & 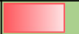 & 3418 & & 0.23 \\
\hline
\end{tabular}

Fig. 3 The top 3 dimensions in each medical context for $E E G$

important. The trained model is then applied to recommend similar terms.

\section{Evaluation}

\section{Medical-context vector space}

We collected notes from the VUMC EMR generated between January 1, 2016, and January 2, 2017. The medical contexts were distributed across a set of dimensions as follows (and shown in Table 1):

(1) Hospital Organizational Structure. 258 departments and 158 types of staff;

(2) Medical Events. 957 ICD-9 codes, 6,537 CPT codes and 11,595 chief complaints in free-text format;

(3) Demographics. Three patient genders (male, female and unknown) and ten age ranges (quantized into ten-year bins up to 100, after which all ages were represented as $100+$ );

(4) Medical Note Structure. 1,514 note types; 61 note sections (defined by the headers as determined by the SecTag method [22]). Five sections ("assessment," "findings," "family medical history," "medica- tions," and "problem list") contain the most important information in a chart review task based on our discussions with the medical researchers.

\section{Datasets}

We created three evaluation datasets associated with chart review tasks (Table 2):

(1) Acute Myocardial Infarction Note Relevance (referred to as the AMI project). This task requires researchers to highlight any portion of a note that contains references to diagnoses, medications, procedures, or symptoms of AMI.

(2) Crohn's Anti-TNF Responsiveness (referred to as the Crohn's project). This task requires researchers to review and highlight text describing whether a patient with Crohn's disease was clinically responsive to anti-TNF medication.

(3) Pediatric Diabetes Note Barriers (referred to as the Diabetes project). This task requires researchers 


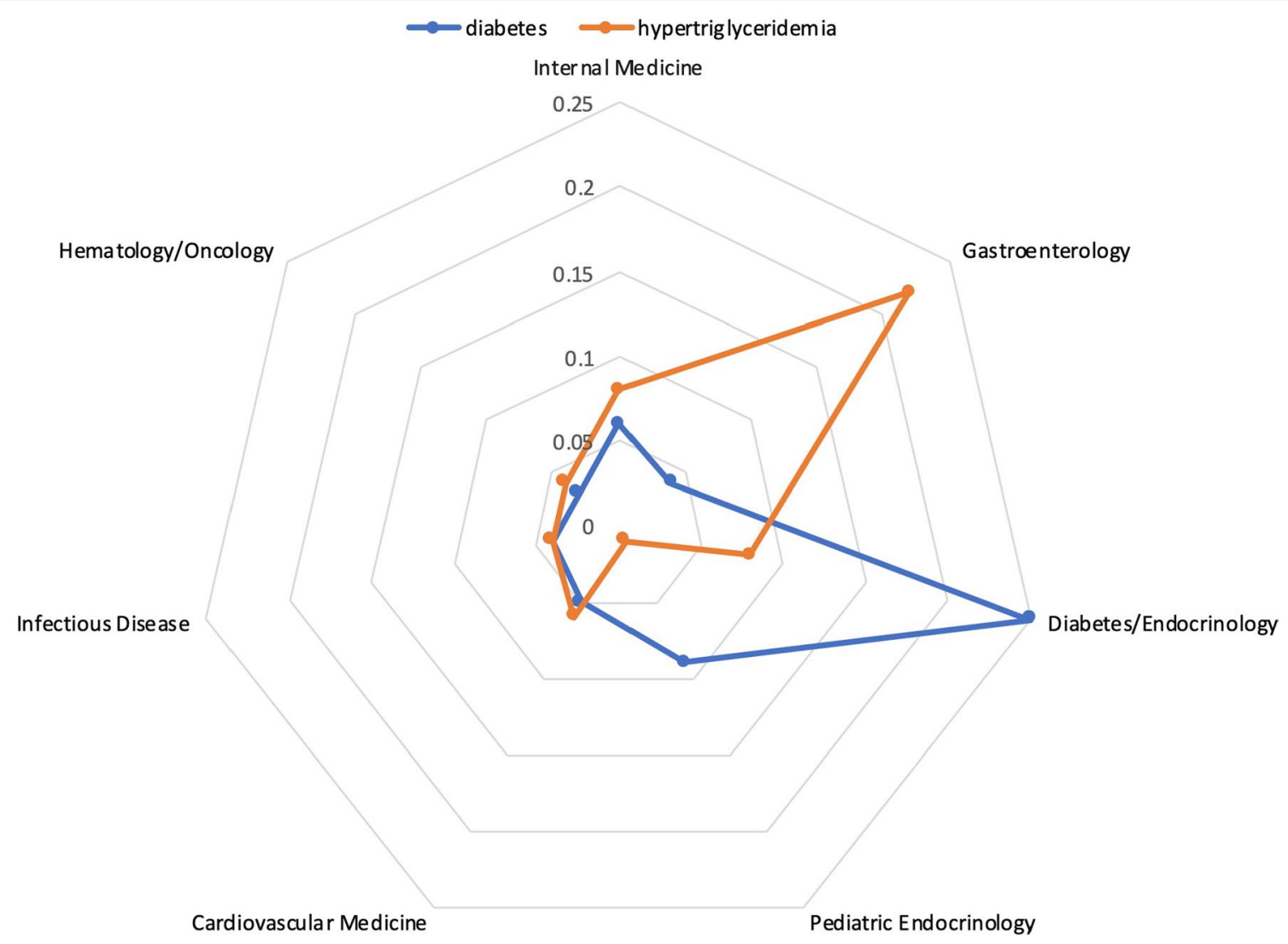

Fig. 4 The medical-context vectors for diabetes and hypertriglyceridemia in the Department medical context

to review a list of medical notes, highlight and label portions of the notes that may be related to barriers in the documentation of diabetes plans.

All of these chart review tasks were deployed in the Vanderbilt's Pybossa crowdsourcing platform [23], and reviewed by chart reviewers who have sufficient medical knowledge. We recruited medical researchers from different disciplines of VUMC, including professors, nurses, and medical students who passed a pre-citification of medical knowledge related to the chart review tasks.

In each of the chart review tasks, the researchers searched and reviewed medical notes to identify and highlight important text snippets for the task. Given the medical notes $D$ of a chart review task $T$, we define the highlighted count $H$ of a clinical term $w$ as the total number of times $w$ is highlighted across all documents in $D$ :

$$
H(w \mid D)=\sum_{d_{j} \in D} H\left(w \mid d_{j}\right)
$$

\section{Experimental design}

We assessed the capabilities of the medical-context vector space and standard word2vec methods by evaluating the extent to which the methods identified the terms that chart reviewers would highlight. Specifically, the term prediction supervised machine learning model is provided highlighted and non-highlighted text as labeled input along with either the medical-context vectors or word2vec vectors, and then predicts if terms will be highlighted. The two hypotheses driving this experiment are: (i) if a term is relevant to a task, then the term should be highlighted by the chart reviewer, and (ii) the terms that are highlighted the most often should be preferred (i.e., predicted by the model) at a higher frequency than nonpreferred terms. The experimental design is as follows.

For each chart review task, a topic word $\mathrm{K}$ is chosen as the most important keyword of the research goal (e.g., diabetes is a topic word of the research task Pediatric Diabetes Note Barriers Problem) and serves as the basis for a similar term generator. Table 2 presents the topic word of each chart review task.

We define the similar terms that might be preferred by the researchers of a chart review task as the 


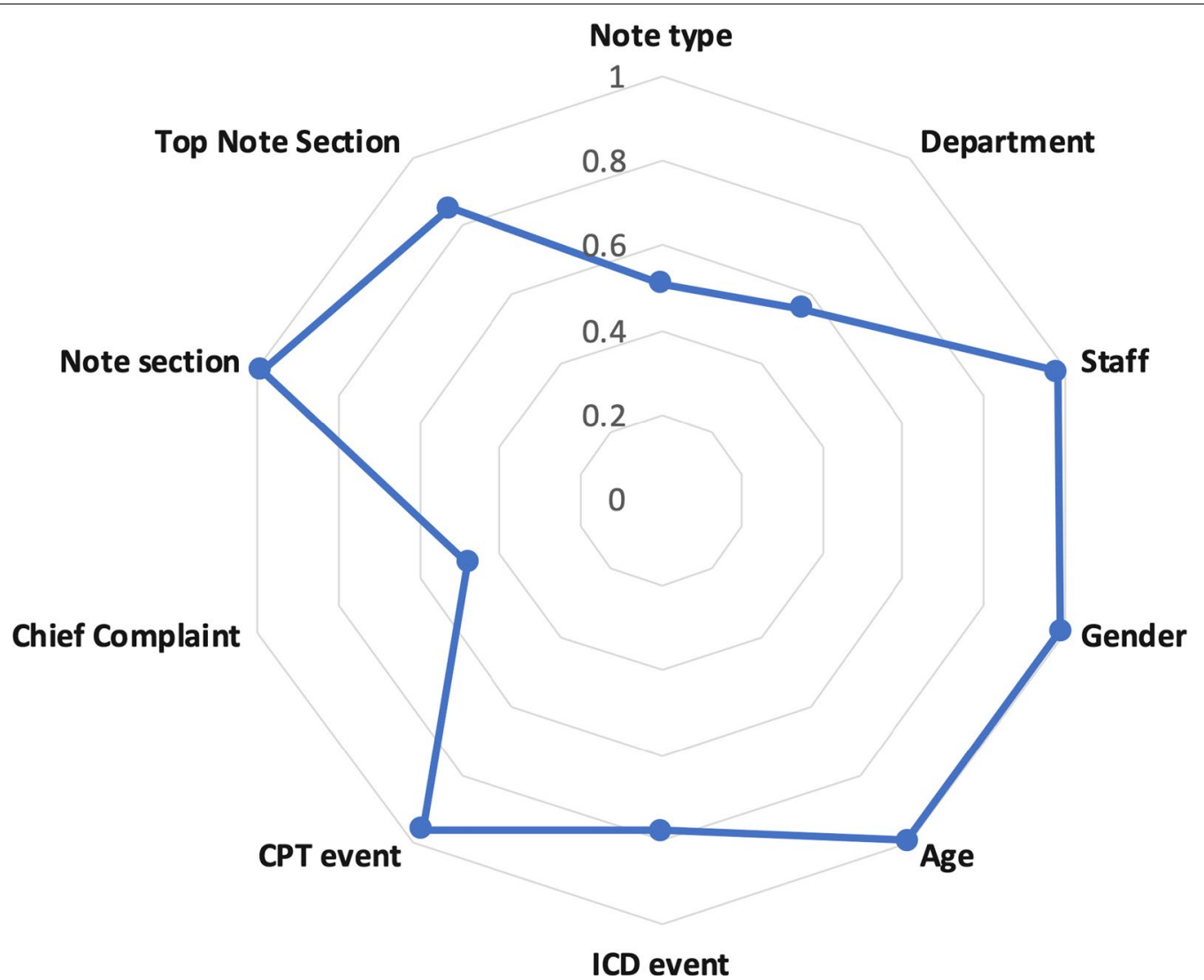

Fig. 5 The medical-context similarities of diabetes and hypertriglyceridemia in all medical contexts

Table 2 Chart review tasks defined for the evaluation

\begin{tabular}{llcr}
\hline Chart review task & Topic word & Patients & Notes \\
\hline Acute myocardial infarction & AMl & 152 & 200 \\
Crohn's anti-TNF Responsiveness & Crohn & 983 & 437,993 \\
pediatric diabetes note barriers & Diabetes & 76 & 210 \\
\hline
\end{tabular}

candidate semantic set. A candidate semantic set $W_{s}$ can be provided by any existing similar term generator, such as EMR-based word2vec embeddings [6, 14, 24, 25], or the EMR-subsets method. A candidate set is used instead of all possible words in the vocabulary as a means to limit the search space.

We define the semantic preference of a chart review task as a subset of preferred similar terms and a subset of non-preferred similar terms from the candidate semantic set. A semantic preference prediction task is formulated as a supervised machine learning task, in which a model learns the semantic preference from a small set of preferred similar terms and non-preferred similar terms (i.e., the training label set). The features of a similar term $w$ are its medical-context similarity vector based on the topic word $K$. The label of a similar term is based on its highlighted count in the chart review task and a given importance cutoff $I$. If the highlighted count of a similar term $\mathrm{w} \in W_{s}$ is greater than $I$, we label it as an important term (i.e., label $=1$ ); otherwise, we label it as a non-important term (i.e., label $=0$ ).

Figure 6 shows an example application of the medical-context vector space to predict the preferred similar terms of reviewers in a chart review task. A classifier based on logistic regression is trained to weight each medical context and obtain the weights of medical context as $W_{c}=\left\{W_{c 1}, W_{c 2}, \ldots, W_{c 10}\right\}$ with a given threshold $I$. Given the medical-context similarity vector $\left\{S_{c 1}(K, w), S_{c 2}(K, w), \ldots, S_{c 10}(K, w)\right\}$ of an unlabeled similar term $t$, the classifier then predicts if an unlabeled similar term $t$ is a preferred similar term of the reviewer and will be highlighted.

\section{Semantic preference prediction evaluation}

This semantic preference prediction task evaluation was performed in the following manner: 


\section{Weight the Medical Contexts}

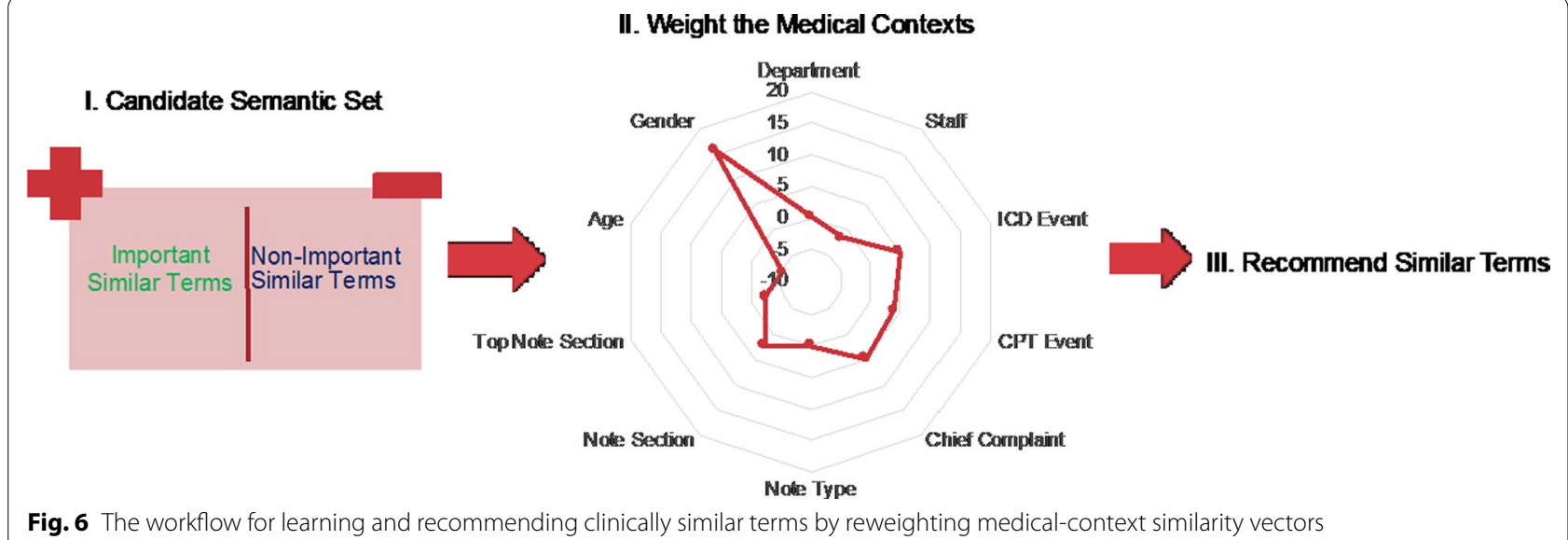

(1) Given an evaluation data set, we first generate a candidate semantic set $W_{s}$ for its topic word using an existing similar term generator.

(2) Given the candidate semantic set $W_{s}$, we construct a label set with an importance cutoff $\mathbf{I}=1$. If the highlighted count of a similar term $w_{i} \in W_{s}$ is greater than 1, we label it as an important term (i.e., label =1); otherwise, we label it as a non-important term (i.e., label value equals 0 ). For each similar term $w_{i}$ in the candidate semantic set $W_{s}$, we generate its medical-context similarity vector $S\left(w_{i}, K\right)$.

(3) We train and evaluate a supervised machine learning model in the label set using ten-fold cross-validation. We evaluated three classifiers: (1) Logistic regression, (2) Random forest, and (3) Support vector machine. We measured the ROC (Receiver Operating Characteristic) curve and reported the AUROC (Area Under the ROC Curve). Other standard metrics (e.g., precision, recall, and F1 score) were considered; however, AUCROC was chosen for its ability to measure the balance of the true positive rate (TPR) and false positive rate (FPR).

(4) We increase the importance cutoff $I$ by 1 and repeat steps (2) and (3) until the number of important terms is less than 10 in the resulting label set. Based on the cross-validation model, we defined 10 as the minimum number of positive labels to ensure each test fold had at least one positive label.

We repeated this process with three similar term generators: (1) the EMR-subsets method [19], (2) the Complete EMR word2vec embedding [19], and (3) the Google News word2vec embedding [16]. The Complete EMR word2vec embedding and the Google News word2vec embedding are also used as baseline feature spaces to recommend similar terms. We use the two baseline embeddings to evaluate if the training data for the word embeddings significantly impacts performance.

\section{Learning curve evaluation}

In a chart review task, the fewer labels required for learning the semantic preference, the earlier we can provide semantic support to reviewers. As such, we further assessed how the size of the training dataset influences the performance of the medical-context vector space. To perform this assessment, we rely on a learning curve analysis [26].

The learning curve analysis task evaluation was performed in the following manner:

(1) Given an evaluation data set, we first generate a candidate semantic set $W_{s}$ using an existing similar term generator.

(2) Given the candidate semantic set $W_{s}$, we constructed a label set with an importance cutoff $I$. When the highlighted count of a similar term $w_{i} \in W_{s}$ is greater than $I$, we label it as an important term (i.e., label $=1$ ), otherwise, we label it as a non-important term (i.e., label value equals 0 ). For each similar term $w_{s i}$ in the candidate semantic set, we generate its medical-context similarity vector $S\left(w_{i}, K\right)$.

(3) Given the label set, we set $x$ to $1 \%$ of the data points as the training set and the remaining $99 \%$ as the test set.

(4) We train a supervised machine learning model with the training set and evaluate its AUROC with the test set. Repeat step (3) and (4) 100 times and measure the AUROC.

(5) Next, we increase $x$ by $1 \%$ and repeat step (3) and (4) until $\mathrm{x}$ is greater than $90 \%$. 
(6) Finally, we increase the importance cutoff $I$ and repeat step (2) to (5) until the number of important terms is less than 10 in the resulting label set.

We repeat this process with the similar term generators used in the Semantic Preference Prediction Experiment.

\section{Interpretable feature space experiment}

Constructing interpretable feature space is essential for medical applications [27, 28], especially chart review tasks. Thus, we assessed the potential of the medicalcontext vector space for providing an interpretable feature space. We applied the binary logistic regression (Eq. 1) to analyze the impacts of medical contexts to reviewers' semantic preference of the three chart review tasks (Table 2) for a term $w$ and the topic word $K$, and interpreted the meanings of the weights of each medical context.

$$
\ln \left(\frac{P(\text { Preferred })}{P(\text { Non-preferred })}\right)=\text { Intercept }+\sum_{i=1}^{10} C_{i} * S_{c_{i}}(w, K)
$$

\section{Results}

\section{Distribution of terms}

Figure 7 shows the distribution of the clinical terms (Keppra, EEG, seizures, epilepsy, and Vimpat) across the note sections. It can be seen that EEG is frequently used in the Assessment/Diagnosis section, while Keppra is more frequently used in the Medications section.

\section{Comparison to BERT}

We compare the medical-context vector's performance to two fine-tuned BERT models: (i) a BERT model finetuned by the approximately four million de-identified prescription notes from the VUMC EMR (which we refer to as the DrugBERT model), and (ii) the pre-trained BioBERT model. We provide the phrase "Keppra medication" to these models, in which medication is the contextual information to help the models contextualize similar terms for Keppra. For the medical-context vector, the phrase Keppra medication is converted into a context vector. For all methods, we find the ten most similar terms.

Table 3 presents the similar terms for Keppra from the models. The result shows that the DrugBERT and BioBERT model provides mostly typos of Keppra (e.g., keppr) as the top similar terms of "Keppra." However, in a clinical chart review task, given the phase Keppra medication, it is more likely the reviewers consider drugs other than Keppra as its top similar terms.

\section{Semantic preference prediction}

Table 4 shows the size of the candidate semantic set provided by the EMR-subsets method and the number of terms highlighted by reviewers. The table shows that for each project, reviewers highlighted different proportions of terms, demonstrating potential variability and challenges for recommending similar terms. In the remainder of this paper, we only show the results based on the candidate semantic sets provided by the EMR-subsets

Table 3 Similar terms for Keppra based on the fine-tuned BERT models and the medical-context vector space

\begin{tabular}{llll}
\hline Similarity rank & DrugBERT & BioBERT & $\begin{array}{l}\text { Medical- } \\
\text { context vector } \\
\text { space }\end{array}$ \\
\hline 1 & keprra & keppr & depakote \\
2 & keppr & onkeppra & vimpat \\
3 & keppera & kepppra & trilepatal \\
4 & keprpa & keppraxr & valproic \\
5 & sezure & keprra & phenobarbital \\
6 & gabatril & prnno & topiramate \\
7 & sezire & ssri & gabatril \\
8 & sizure & andmri & lamictal \\
9 & seizre & najib & fosphenytoin \\
10 & equetro & nimotop & zonisamide \\
\hline
\end{tabular}

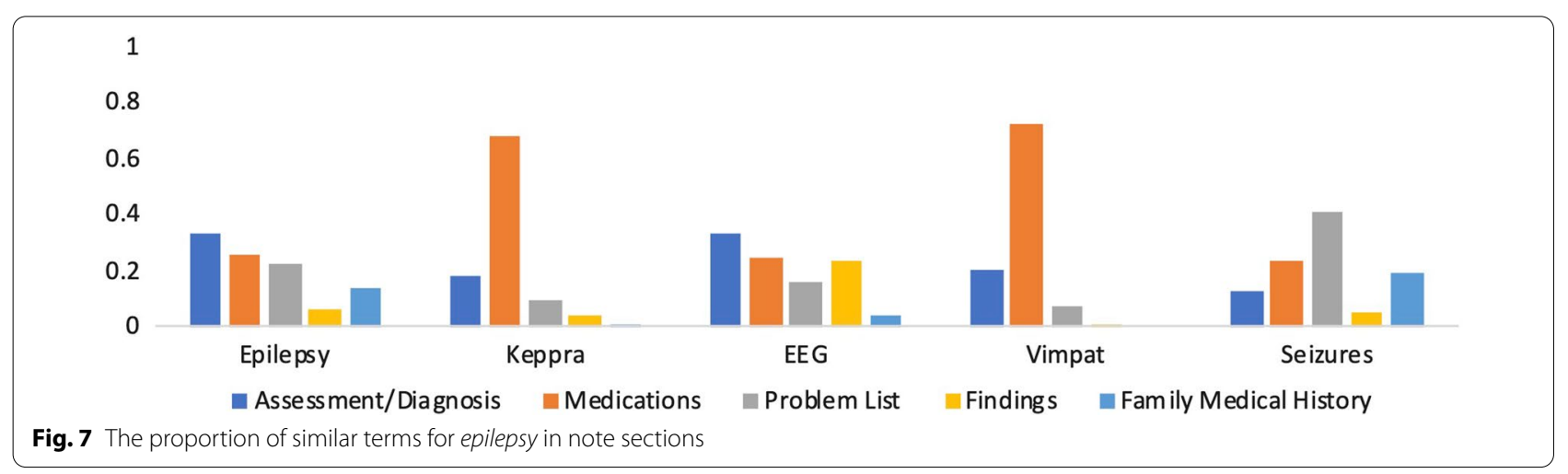


Table 4 The candidate semantic sets of the chart review tasks

\begin{tabular}{lll}
\hline Dataset & Candidate similar terms & $\begin{array}{l}\text { Unique } \\
\text { highlighted } \\
\text { similar terms }\end{array}$ \\
\hline AMl & 1949 & 1414 \\
Crohn & 1204 & 438 \\
Diabetes & 1055 & 273 \\
\hline
\end{tabular}

Table 5 Diabetes dataset average ROC AUROC scores with an importance cutoff of 10

\begin{tabular}{lll}
\hline Model & AUROC & \\
\cline { 2 - 3 } & $\begin{array}{l}\text { Medical-context vector } \\
\text { space features }\end{array}$ & $\begin{array}{l}\text { word2vec } \\
\text { features }\end{array}$ \\
\hline Logistic regression & $0.80^{*}$ & 0.58 \\
Random forest & $0.68^{*}$ & 0.54 \\
Support vector machine & $0.78^{*}$ & 0.57 \\
\hline
\end{tabular}

${ }^{*} \mathrm{p}<0.05$

Table 6 AMl dataset average ROC AUROC scores with an importance cutoff of 40

\begin{tabular}{lll}
\hline Model & AUROC & \\
\cline { 2 - 3 } & $\begin{array}{l}\text { Medical-context vector } \\
\text { space features }\end{array}$ & $\begin{array}{l}\text { word2vec } \\
\text { features }\end{array}$ \\
\hline Logistic regression & $0.80^{* *}$ & 0.73 \\
Random forest & $0.75^{* * *}$ & 0.56 \\
Support vector machine & $0.75^{*}$ & 0.71 \\
\hline$* * \mathrm{p}<0.001, * * \mathrm{p}<0.01, * \mathrm{p}<0.05$ & &
\end{tabular}

Table 7 Crohn dataset average ROC AUROC scores with an importance cutoff of 1

\begin{tabular}{lll}
\hline Model & AUROC & \\
\cline { 2 - 3 } & $\begin{array}{l}\text { Medical-context vector } \\
\text { space features }\end{array}$ & $\begin{array}{l}\text { word2vec } \\
\text { features }\end{array}$ \\
\hline Logistic regression & $0.79^{* *}$ & 0.68 \\
Random forest & $0.80^{* * *}$ & 0.60 \\
Support vector machine & $0.79^{* * *}$ & 0.68 \\
\hline
\end{tabular}

${ }^{* * *} p<0.001,{ }^{* *} p<0.01,{ }^{*} p<0.05$

method and the baseline Complete EMR word2vec embedding because the Google New embedding resulted in similar results to the EMR embedding.

The medical-context vector space's AUCROC outperformed all baseline word2vec embeddings in all evaluation datasets across all similar term generators. Tables 5 , 6 and 7 provide the three example comparisons of the medical-context vector space and the baseline Complete EMR word2vec embeddings for three datasets.
A one-sided Mann-Whitney $U$ test indicated that the medical-context vector space statistically significantly outperformed the baseline Complete EMR word2vec embedding.

Figure 8 shows the result of the Semantic Preference Prediction evaluation using the Diabetes dataset and the candidate semantic set generated by the EMR-subsets method [19].

\section{Learning curve analysis}

As shown in Fig. 9, the medical-context vector space outperformed the EMR-based word2vec embedding regardless of the size of the training data set. It can be seen that the medical-context vector space significantly reduces the number of required labels for learning the semantic preference. For example, as shown in Fig. 9, with only 1\% of the label set, the medical-context vector space reached an AUROC of 0.7 while the baseline Complete EMR word2vec embedding only achieved 0.5 . When using $10 \%$ of the labels, the medical-context vector space and word2vect achieved an AUROC of 0.78 and 0.60 , respectively.

\section{Interpretable feature space}

As shown in Table 8, the Chief Complaint medical context has a significant positive impact on reviewers' semantic preference, which means the clinical terms that are similar in describing the same chief complaint of a chart review task are preferred by the reviewers. It is interesting that the Gender context had the highest significant positive impact on the semantic preference of the AMI chart review task. Since the topic word $A M I$ likely has little relevance with respect to gender, terms highly relevant to gender were not preferred by the reviewers.

\section{Discussion}

This paper presents a novel vector space model, the medical-context vector space, to identify similar terms to support chart reviews. The medical-context vector space is a collection of normalized-frequencies of clinical terms in different medical contexts, which provide information on the relationships between clinical terms. We evaluated the medical-context vector space for predicting the preferred similar terms of reviewers in three chart review tasks. The results show that the medical-context vector space efficiently learned the preferred similar terms of reviewers and outperformed the baseline word2vec embedding in all three chart review tasks as measured with the AUROC metric. Additionally, the medicalcontext vector space significantly reduced the number of labels (e.g., from thousands of labels to tens of labels) required to learn and predict the preferred similar terms of reviewers. 


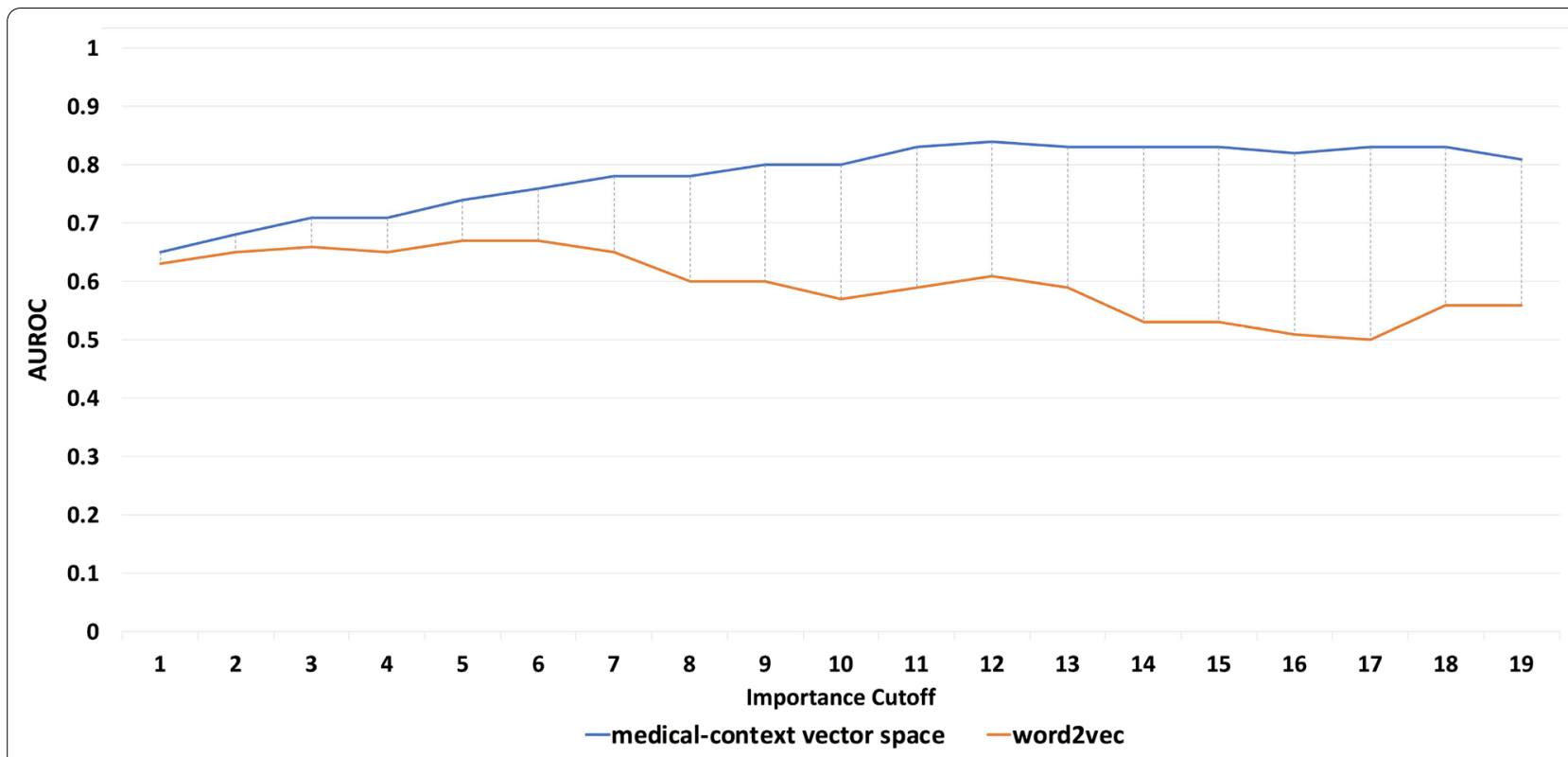

Fig. 8 Average AUROC achieved by the logistic regression classifier for the diabetes dataset

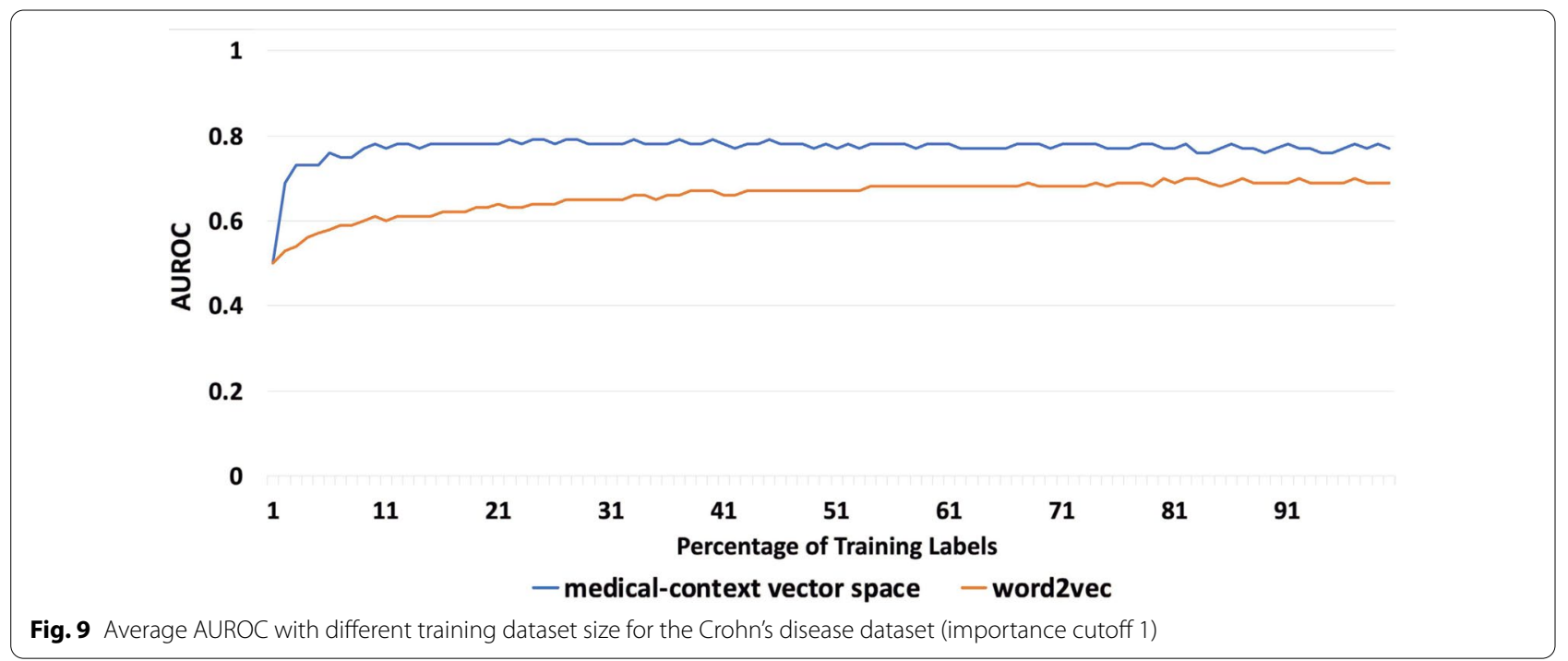

There are several possible reasons why the medicalcontext vector space outperformed the baseline methods. First, the feature space provided by the medical-context vector space is much smaller than the feature space provided by the word2vec embedding (i.e., 10 dimensions vs. 100 dimensions of the Complete EMR word2vec embedding). Second, the feature space provided by the medical-context vector space is more capable of capturing relationships between terms induced by external, non-textual forces. For instance, context such as the chief complaint, the author's department and the patient's age influence the terms a chart reviewer prefers for a given task, yet these factors are not captured in traditional word embedding models. Third, the construction of medical contexts (e.g., note sections), and counting the frequency of words in those contexts, implicitly captures relationships between terms in structured ways that otherwise would be difficult to extract based on the text alone.

The medical-context vector adjusts to the reviewer's desired semantics by eliciting reviewer input. Previous research has similarly demonstrated that clinical natural language processing models (e.g., word sense disambiguation) can be trained by asking experts to provide 
Table 8 The impact of medical contexts on reviewers' preference in AMI task

\begin{tabular}{llccc}
\hline Index & Context & \multicolumn{2}{c}{ Coefficient } & \\
\cline { 3 - 5 } & & AMI & Crohn's & Diabetes \\
\hline 1 & Intercept & $-16.16^{* * *}$ & $-13.82^{* * *}$ & -22.04 \\
2 & Department & 0.58 & 0.42 & 0.33 \\
3 & Staff & 1.90 & 0.64 & -1.49 \\
4 & ICD event & $-2.94^{* *}$ & $2.18^{* *}$ & 4.89 \\
5 & CPT event & 0.37 & $1.35^{*}$ & 4.10 \\
6 & Chief complaint & $5.75^{* * *}$ & $7.24^{* * *}$ & $4.93^{* * *}$ \\
7 & Note type & $5.70^{* * *}$ & $-1.92^{*}$ & -0.05 \\
8 & Note section & $2.00^{* * *}$ & -0.23 & $2.37^{* *}$ \\
9 & Top five note sections & 1.43 & $1.37^{*}$ & -2.54 \\
10 & Age & 0.37 & $2.65^{* * *}$ & $-5.41^{* * *}$ \\
11 & Gender & $8.85^{* * *}$ & $8.03^{* * *}$ & 15.78 \\
\hline
\end{tabular}

${ }^{* * *} p<0.001,{ }^{* *} p<0.01,{ }^{*} p<0.05$, one-tailed

labeled instances $[29,30]$. This iterative process allows the medical-context vector to essentially be fine-tuned for the specific task. While other fine-tuning methods have been proposed and shown to be successful using text, the fine-tuning process used in this work relies on the non-textual contexts that are encoded. This explicit encoding of context allows for rapid learning of the reviewer's preference, as demonstrated by the number of labels needed from reviewers.

Fine-tuned BERT models, such as the DrugBERT and BioBERT, can be tuned for a specific chart review task. However, this study shows that the resulting embeddings recommend terms in different ways than the medicalcontext vector. For example, when looking for similar terms of a seizure drug, BERT fine-tuned models recommend typos or misspellings, while the medical-context vector recommends other drugs with the same clinical purpose. Thus, while BERT and fine-tuned BERT can be useful for expanding terms for clinical chart review tasks, the medical-context vector fills in an essential technological gap when identifying similar terms based on the context in which terms are used.

In this study, three machine learning methods (namely, logistic regression, random forest, and support vector machines) were evaluated to determine how well they predict the preferred terms for clinically knowledgeable reviewers.

All three machine learning models attained better performance when using the medical-context vector space compared to the word2vec embeddings. In a follow-up pilot study, several clinical researchers were invited to test a prototype user interface (Additional file 1: Figure D). Their feedback suggested that the logistic regression method might be preferred by clinical researchers because the results are both accurate and easily interpretable by examining the weights of the logistic coefficients).

By contrast, methods based on random forests and support vector machines can be more difficult to interpret due to the complexity of the models. Specifically, random forests learn a list of features, which creates subgroups of variables, and then builds an ensemble over the subgroups. Support vector machines identify a decision surface in a feature space that is higher in dimensionality than the original system to separate different classes.

There are several limitations of this study that highlight opportunities for future research. First, when building the medical-context vector space, we limited the time range used to build the medical event context of a note to $48 \mathrm{~h}$ (i.e., a chief complaint had to be within $48 \mathrm{~h}$ of when the note was written). It is unknown if different time ranges would impact these findings. Second, in this study, we focused on ten medical contexts when building the medical-context vector space, but there are clearly others that could be considered. Third, this pilot study indicated that certain medical contexts (e.g., the "Chief Complaint" medical context) have a significant impact on reviewers' semantic preferences in a chart review task, but it is necessary to survey reviewers to learn why they prefer such terms.

To refine this system, the vector space could be enhanced in several ways. First, domain knowledge could be introduced by adding more knowledge-based dimensions, such as a laboratory result dimension, which would be oriented to capture how providers use words when describing test values. Second, the system could be extended by inviting clinical researchers to identify the important medical contexts. As shown in Additional file 1: Figure D, a clinical researcher selects words from a candidate word list (left column of the UI) and drag each word into the positive (negative) area if they are preferred (or not). The system will learn the preferred contexts of clinical researchers and identify the most important medical contexts.

The vector space method can be put into practice and further tested in our existing EMR search engine (Additional file 1: Figure E) [19, 23]. The search engine takes a keyword as input which is expanded to a set of terms used for document retrieval based on the vector space.

\section{Conclusions}

In this paper, we presented a novel vector space model, the medical-context vector space, to represent how clinical terms were used in varying medical situations. We evaluated the performance of the medical-context vector space in predicting the preferred similar terms of reviewers in three chart review tasks. The empirical findings show that the medical-context vector space achieved good 
performance and significantly outperforms baseline word2 vec embeddings. Additionally, the medical-context vector space significantly reduced the number of labels required to learn and predict the preferred similar terms of reviewers. This research suggests that the medical-context vector space can better identify preferred similar terms based on non-textual features compared to traditional word embedding models.

\section{Supplementary Information}

The online version contains supplementary material available at https://doi. org/10.1186/s12911-021-01724-2.

Additional file 1. Supplemental Appendix

\section{Acknowledgements}

The data for this study was based on the VUMC data repositories, which are supported by institutional funding and by the Vanderbilt CTSA Grant ULTR000445 from NCATS/NIH.

\section{Author contributions}

$\mathrm{CY}$ and DF proposed and formulated the problem, recruited clinical researchers, collected the data, developed and evaluated the methods, and composed the manuscript. BM interpreted the experimental results and assisted in the writing and revision of the manuscript. All authors read and approved the final manuscript.

\section{Funding}

Crowd Sourcing Labels from Electronic Medical Records to Enable Biomedical Research Award Number: 1 UH2 CA203708-01.

\section{Availability of data and materials}

The data for this study was based on the VUMC data repositories. The data are available within VUMC but restrictions are applied to the public availability of these data, and so are not publicly available. Data are however available from the corresponding author upon reasonable request and with the permission of VUMC.

\section{Declarations}

Ethics approval and consent to participate

The study was approved by the Vanderbilt University Institutional Review Board (\#160995 and \#140917).

\section{Consent for publication}

Not applicable.

\section{Competing interests}

The authors declare that they have no competing interests.

\section{Author details}

'Department of Computer Science, Vanderbilt University, 2301 Vanderbilt Place, PMB 351679, Nashville, TN 37235-1679, USA. ${ }^{2}$ Department of Biomedical Informatics, Vanderbilt University Medical Center, Nashville, TN, USA. ${ }^{3}$ Department of Biostatistics, Vanderbilt University Medical Center, Nashville, TN, USA.

Received: 19 January 2021 Accepted: 9 December 2021

Published online: 18 December 2021

\section{References}

1. Beers LS, Godoy L, John T, Long M, Biel MG, Anthony B, et al. Mental health screening quality improvement learning collaborative in pediatric primary care. Pediatrics. 2017;140(6):e20162966.
2. Rasmussen $L V$. The electronic health record for translational research. J Cardiovasc Transl Res. 2014 [cited 2017 Jun 23];7(6):607-14.

3. Chen L, Guo U, Illipparambil LC, Netherton MD, Sheshadri B, Karu E, et al. Racing against the clock: internal medicine residents'time spent on electronic health records. J Grad Med Educ. 2016 [cited 2017 Apr 19];8(1):39-44. https://doi.org/10.4300/JGME-D-15-00240.1.

4. Hripcsak G, Vawdrey DK, Fred MR, Bostwick SB. Use of electronic clinical documentation: time spent and team interactions. J Am Med Inf Assoc. 2011;18(2):112-7.

5. Ooi J, Ma X, Qin H, Liew SC. A survey of query expansion, query suggestion and query refinement techniques. In: 2015 4th international conference on software engineering and computer systems ICSECS 2015 Virtuous Software Solutions Big Data. 2015; p. 112-7.

6. Goodwin T, Harabagiu SM. UTD at TREC 2014: query expansion for clinical decision support. 23rd text retrieval conference (TREC 2014) Proceedings. 2014;1.

7. Pal $\mathrm{D}$, Mitra $\mathrm{M}, \mathrm{Bhattacharya} \mathrm{S.} \mathrm{Exploring} \mathrm{query} \mathrm{categorisation} \mathrm{for} \mathrm{query}$ expansion: a study. 2015;1-34. arXiv:1509.05567.

8. Wang Y, Liu S, Afzal N, Rastegar-Mojarad M, Wang L, Shen F, et al. A comparison of word embeddings for the biomedical natural language processing. J Biomed Inform. 2018;87(September):12-20. https://doi.org/ 10.1016/j.jbi.2018.09.008

9. Biron P, Metzger MH, Pezet C, Sebban C, Barthuet E, Durand T. An information retrieval system for computerized patient records in the context of a daily hospital practice: the example of the Léon Bérard Cancer Center (France). Appl Clin Inform. 2014 [cited 2016 Oct 24];5(1):191-205. https:// doi.org/10.4338/ACl-2013-08-CR-0065.

10. Natarajan $K$, Stein $D$, Jain S, Elhadad N. An analysis of clinical queries in an electronic health record search utility. Int J Med Inform. 2010 [cited 2016 Oct 24];79(7):515-22.

11. Tawfik AA, Kochendorfer KM, Saparova D, Al Ghenaimi S, Moore JL."I don't have time to dig back through this": the role of semantic search in supporting physician information seeking in an electronic health record. Perform Improv Q. 2014 [cited 2016 Oct 24];26(4):75-91. https://doi.org/ 10.1002/piq.21158.

12. Zalis M, Harris M. Advanced search of the electronic medical record: Augmenting safety and efficiency in radiology. J Am Coll Radiol. 2010 [cited 2016 Oct 24];7: 625-33.

13. Gregg W, Jirjis J, Lorenzi NM, Giuse D. StarTracker: an integrated, webbased clinical search engine. AMIA Annu Symp Proc. 2003 [cited 2016 Oct 24];2003(1):855.

14. Hanauer DA, Mei Q, Law J, Khanna R, Zheng K. Supporting information retrieval from electronic health records: a report of University of Michigan's nine-year experience in developing and using the Electronic Medical Record Search Engine (EMERSE). J Biomed Inform. 2015;55:290-300. https://doi.org/10.1016/j.jbi.2015.05.003.

15. Mikolov T, Chen K, Corrado G, Dean J. Distributed representations of words and phrases and their compositionality. Adv Neural Inf Process Syst. 2013;3111-9.

16. Mikolov T, Corrado G, Chen K, Dean J. Efficient estimation of word representations in vector space. In: Proceedings of International Conference on Learning Representations (ICLR 2013). 2013; p. 1-12.

17. Devlin J, Chang M-W, Lee K, Toutanova K. BERT: pre-training of deep bidirectional transformers for language understanding. 2018. arXiv:1810. 04805.

18. Li F, Jin Y, Liu W, Rawat BPS, Cai P, Yu H. Fine-tuning bidirectional encoder representations from transformers (BERT)-based models on large-scale electronic health record notes: an empirical study. J Med Internet Res. 2019;21(9):1-13.

19. Ye C, Fabbri D. Extracting similar terms from multiple EMR-based semantic embeddings to support chart reviews. J Biomed Inform. 2018;83(April).

20. Lee J, Yoon W, Kim S, Kim D, Kim S, So CH, et al. BioBERT: a pre-trained biomedical language representation model for biomedical text mining. Bioinformatics. 2020;36(4):1234-40. https://doi.org/10.1093/bioinforma tics/btz682.

21. Mitchell MJ, King MR. Secondary use of clinical data: the vanderbilt approach. J Biomed Inform. 2014;52:28-35.

22. Denny JC, Spickard A, Johnson KB, Peterson NB, Peterson JF, Miller RA Evaluation of a method to identify and categorize section headers in clinical documents. J Am Med Informatics Assoc. 2009;16(6):806-15. 
23. Ye C, Coco J, Epishova A, Hajaj C, Bogardus H, Novak L, et al. A crowdsourcing framework for medical data sets. AMIA Summits Transl Sci Proc. 2018;2017:273-80.

24. Martinez D, Otegi A, Soroa A, Agirre E. Improving search over Electronic Health Records using UMLS-based query expansion through random walks. J Biomed Inform. 2014;51:100-6. https://doi.org/10.1016/j.jbi.2014. 04.013.

25. Malhotra A, Younesi E, Gündel M, Müller B, Heneka MT, Hofmann-Apitius M. ADO: a disease ontology representing the domain knowledge specific to Alzheimer's disease. Alzheimer's Dement. 2014;10(2):238-46.

26. Perlich C, Provost F, Simonoff JS. Tree induction vs. logistic regression: a learning-curve analysis. J Mach Learn Res. 2003;4:211-55.

27. Ribeiro MT, Singh S, Guestrin C. "Why should I trust you?": explaining the predictions of any classifier. In: Proceedings of the 22nd ACM SIGKDD international conference on knowledge discovery and data mining. ACM; 2016. p. 1135-44. arXiv:1602.04938.

28. Temple MW, Lehmann CU, Fabbri D. Predicting discharge dates From the NICU using progress note data. Pediatrics. 2015 [cited 2017 Aug 16];136(2):e395-405.

29. Wang Y, Zheng K, Xu H, Mei Q. Interactive medical word sense disambiguation through informed learning. J Am Med Informatics Assoc. 2018;25(7):800-8. https://doi.org/10.1093/jamia/ocy013.

30. Self JZ, Vinayagam RK, Fry JT, North C. Bridging the gap between user intention and model parameters for human-in-the-loop data analytics. In: HILDA@ SIGMOD. 2016.p.1-6.

\section{Publisher's Note}

Springer Nature remains neutral with regard to jurisdictional claims in published maps and institutional affiliations.

- fast, convenient online submission

- thorough peer review by experienced researchers in your field

- rapid publication on acceptance

- support for research data, including large and complex data types

- gold Open Access which fosters wider collaboration and increased citations

- maximum visibility for your research: over $100 \mathrm{M}$ website views per year

At BMC, research is always in progress.

Learn more biomedcentral.com/submissions 